\title{
Pan-Genomic Analysis of African Swine Fever Virus
}

\author{
Ziming Wang ${ }^{1,2,3} \cdot$ Lijia Jia $^{1,2,3} \cdot$ Jing $\mathrm{Li}^{3,4} \cdot$ Haizhou Liu ${ }^{2} \cdot \mathrm{Di} \mathrm{Liu}^{1,2,3,5,6}$ (D)
}

Received: 10 September 2019/Accepted: 17 October 2019/Published online: 11 December 2019

(C) The Author(s) 2019

\section{Dear Editor,}

African swine fever (ASF) is a severe haemorrhagic fever in domestic pigs and wild boar with extremely high mortality rate. It is cataloged as a notifiable disease by the World Organization for Animal Health (OIE). The etiological agent that causes the highly lethal disease is the African swine fever virus (ASFV) (Sanchez-Vizcaino et al. 2015). ASFV is the only known member of the genus Asfivirus and family Asfarviridae. The family Asfarviridae belongs to the member of nucleocytoplasmic large DNA viruses (NCLDV) superfamily (Iyer et al. 2006; Costard et al. 2009). Overall, the ASFV virion presents an icosahedral morphology with a multilayered structure (Wang et al. 2019). The genome of ASFV is a large doublestranded DNA (dsDNA) molecule that varies in length from about 170 to 193 kilobase pairs and encodes between 150 and 167 open reading frames (ORFs) depending on the isolate (Dixon et al. 2013). In addition, ASFV also infects

Electronic supplementary material The online version of this article (https://doi.org/10.1007/s12250-019-00173-6) contains supplementary material, which is available to authorized users.

\section{Di Liu}

liud@wh.iov.cn

1 CAS Key Laboratory of Special Pathogens and Biosafety, Wuhan Institute of Virology, Chinese Academy of Sciences, Wuhan 430071, China

2 Computational Virology Group, Center for Bacteria and Virus Resources and Bioinformation, Wuhan Institute of Virology, Chinese Academy of Sciences, Wuhan 430071, China

3 University of Chinese Academy of Sciences, Beijing 100049, China

4 CAS Key Laboratory of Pathogenic Microbiology and Immunology, Institute of Microbiology, Chinese Academy of Sciences, Beijing 100101, China

5 African Swine Fever Regional Laboratory of China, Wuhan 430071, China

6 Center for Biosafety Mega-Science, Chinese Academy of Sciences, Wuhan 430071, China
African wild suids, including warthogs (Phacochoerus africanus) and bushpigs (Potamochoerus larvatus), which act as asymptomatic carriers. Soft ticks of the Ornithodoros moubata complex also serve as a natural reservoir and transmit the disease to suids. In East Africa, ASFV is maintained in an ancient sylvatic cycle involving warthogs and soft ticks (Ornithodoros genus) that inhabit their burrows (Jori et al. 2013).

ASF was first reported in Kenya in 1921, and now the disease has been endemic for more than twenty subSaharan African countries. In August 2018, the first ASF outbreak in China (Shenyang, Liaoning Province) was reported to the OIE. Other Asian countries, such as Mongolia, Cambodia, Vietnam, and North Korea, have reported ASF outbreaks in 2019 (Dixon et al. 2019). So far, there is no effective vaccine or antiviral strategy available against ASF. Previous research has shown that variation between the genomes of diverse ASFV isolates results from gain or loss of members of multigene families (MGFs). The families include MGF 100, 110, 300, 360 and 505/530. Additionally, family p22, encoding an early membrane protein, which is adjacent to the left genome terminus exist in some isolates in 1 or 2 copies close to the right genome end (Chapman et al. 2008; Dixon et al. 2013). Nevertheless, it is still enigmatic which of these genes are requisite and which are secondary, and which are in charge of generality and characteristic during evolution.

Currently, the rapid development of high-throughput next-generation sequencing technologies has facilitated the genomics research. Up to now, more than forty ASFV genome sequences of different origin and virulence are available in public databases. Pan-genomic analysis provides a cogent way to estimate the genomic generality and individuality of ASFV strains, and to extrapolate the number of additional whole genomes sequences that would be necessary to characterize the overall pan-genome or gene repertoire. The term pan-genome used to describe the entire repertoire of genes or ORFs shared by genomes of interest, including the core genome that contains genes shared by all strains and the dispensable genome made of genes shared by a subset of the strains, and strain-specific 
A

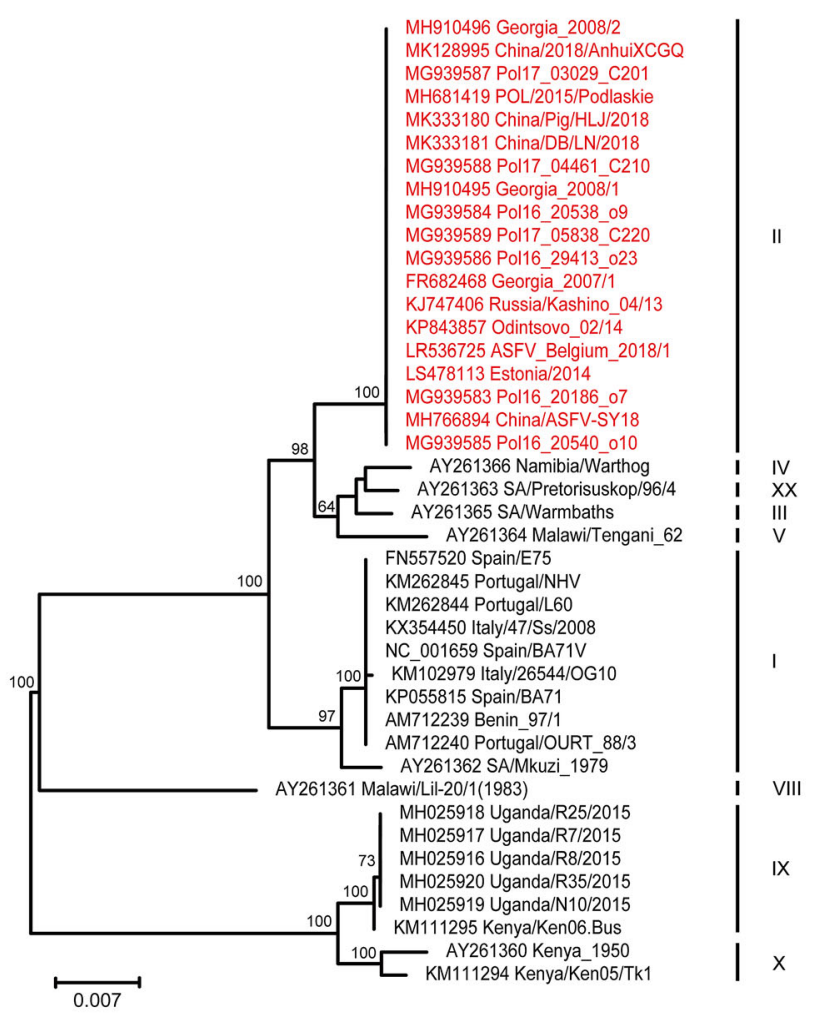

B

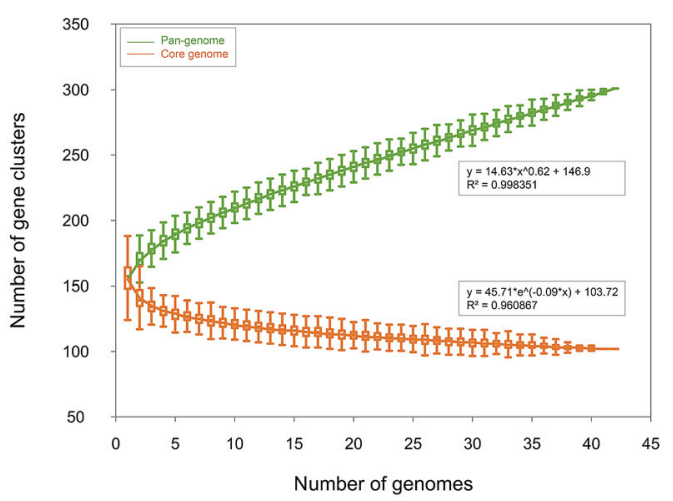

C

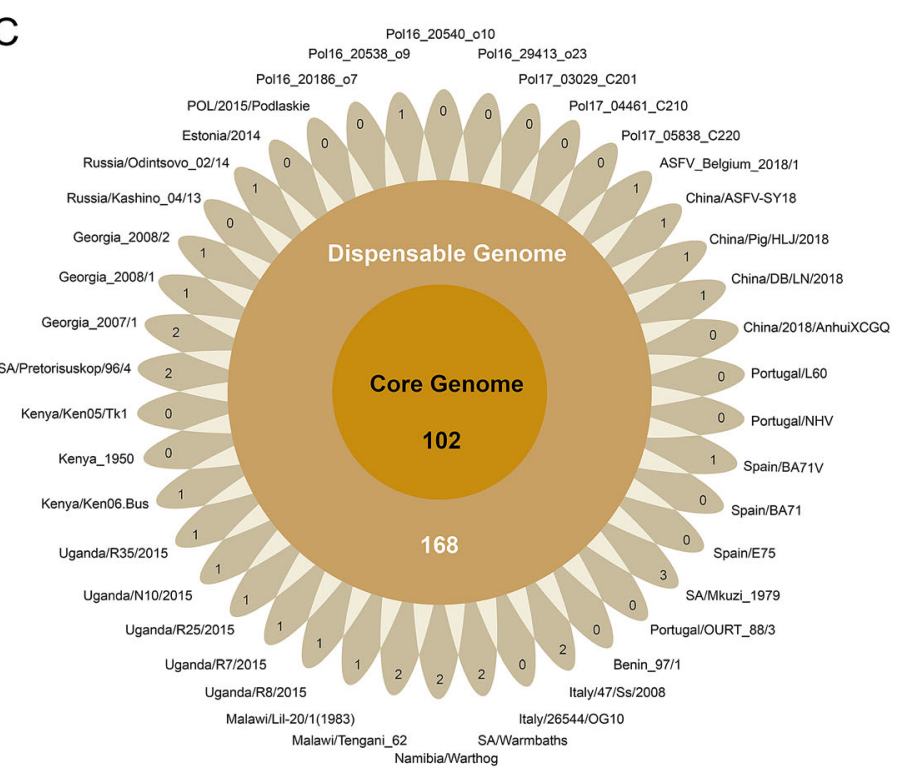

zero, indicating an open pan-genome. The curve is the least squares fit of the power law to the average values. C Flower plot painting the core genome, dispensable genome, and strain-specific genes of the 42 ASFV strains. The diagram depicts the core gene number (in the center), the dispensable gene number (in the annulus), and the strainspecific gene number (in the petals) for the 42 ASFV strains.

determining the partially shared and strain-specific characteristics of a species that are not essential to its basic lifestyle (Vernikos et al. 2015). 
Here, we conducted pan-genome analysis of 42 genomes of ASFV available in GenBank to understand their genomic peculiarity. Firstly, a total of forty-two ASFV genome sequences used in this analysis were retrieved from NCBI GenBank database. The analyzed ASFV genomes were mainly isolated from Africa and Europe, and the avirulent BA71V strain which adapted to proliferate in Vero cells and four isolates from China were also included (Bao et al. 2019; Wen et al. 2019). These isolates involved a long period of time between 1950 and 2018, and their host mostly cover domestic pigs, wild boars and ticks. The length of entire genomes varies from 170,101 bp to 193,886 bp (GC contents from $38.0 \%$ to $38.9 \%$ ), averaging at $186,817 \mathrm{bp}$. The majority of isolates possess high virulence, yet the virulence of a few isolates is currently unknown. Viral genomes were annotated using the Genome Annotation Transfer Utility (GATU) (Tcherepanov et al. 2006) with the default parameters. The GATU detects all the potential ORFs present in the target and automatically annotates the unknown sequences by using a reference genome.

In previous studies, twenty-four genotypes have been depicted, with the major genotype groups identified based on the C-terminal end of the B646L gene coding for the capsid protein p72 (Bastos et al. 2003). To determine the genotype of all analyzed strains, the MUSCLE v3.8.31 (Edgar 2004) was employed to align the partial nucleotide sequences of $p 72$ gene from all strains. Phylogenies were inferred using the maximum-likelihood algorithm in RAxML v8.2.12 (Stamatakis 2014) with 1000 bootstrap replicates. The final phylogenetic tree was visualized using FigTree v1.4.4 (http://tree.bio.ed.ac.uk/software/figtree/). The phylogenetic analysis based on partial p72 gene sequences revealed that all the $42 \mathrm{ASFV}$ strains were grouped into nine genotypes (Fig. 1A). Notably, the most widely spread genotype was genotype II. This genotype accounted for more than $40 \%$ of all ASFV strains, including four isolates circulating in China. The GenBank accession number, strain name, country of isolation, year of isolation, and other features are listed in Supplementary Table S1.

The pan- and core genome analyses of the 42 ASFV genomes were performed using PGAP v1.2.1 (Zhao et al. 2012) with Gene Family (GF) method. All protein sequences were aligned using blastall with default parameters (e-value: $1 \mathrm{e}^{-10}$; identity: 0.5 ; coverage: 0.5 ; score: 40), and ortholog clusters were organized using MCL program. Heaps' Law model was employed to fit the pangenome size of strains, and exponential model was applied to fit the core genome size. At last, the characteristic curves of the ASFV pan-genome, the core genome, and the new genes were portrayed using PanGP (Zhao et al. 2014) with DG sampling algorithms. Overall, 301 ortholog clusters were identified, which constituted the ASFV pan-genome. The mathematical function was delineated on the graph which shows an exponential value over 0.5 , indicating the pan-genome of ASFV is in an open state (Fig. 1B). This trend reflects that ASFV has flexible genome contents, and the size of pan-genome may expand with each added genome which contributes to new genes. As the number of analyzed genomes increases, the core genome curve presents a converging trend. Eventually the number of core genome tends to become a stable value, encompassing 102 ortholog clusters in the core genome (Fig. 1C). The core genome mainly encodes structural proteins, enzymes required for replication and transcription, and factors involved in evading host defense systems and coordinating host cell function. The currently known functions of encoding genes that belong to the core genome are listed in Supplementary Table S2. Furthermore, the ASFV pangenome also contains 168 dispensable genes and 31 strainspecific genes. These unnecessary genes are deemed to have endowed ASFV with a series of selective advantages in the corresponding environmental niche and the ability to colonize new hosts, resulting in the individuality and diversity of ASFV genome.

To sum up, the investigation revealed the pan-genome of ASFV presented an open state and the core genome was conserved in all of the analyzed strains. An open pangenome is typical of microorganisms that colonize multiple environments and have multifarious ways of exchanging genetic material (Medini et al. 2005). Recent studies have also shown that the homologous recombination contributed much to the genetic diversity of ASFVs (Zhu et al. 2019). Illustration of the core genome confers a fundamental understanding of the conservation to ASFV genome during evolution. Our analysis provides a brand-new view on the genomic diversity of ASFV, accelerating our comprehensive understanding of this species.

Acknowledgements This work was supported by grants from the National Key Research and Development Program of China (2018YFC1603803, 2018YFC0840402 and 2016YFC1200800) and the Research Project of African Swine Fever of Chinese Academy of Sciences (KJZD-SW-L06).

\section{Compliance with Ethical Standards}

Conflict of interest The authors declare that they have no conflict of interest.

Animal and Human Rights Statement This article does not contain any studies with human or animal subjects performed by any of the authors.

Open Access This article is distributed under the terms of the Creative Commons Attribution 4.0 International License (http://creative commons.org/licenses/by/4.0/), which permits unrestricted use, distribution, and reproduction in any medium, provided you give 
appropriate credit to the original author(s) and the source, provide a link to the Creative Commons license, and indicate if changes were made.

\section{References}

Bao JY, Wang QH, Lin P, Liu CJ, Li L, Wu XD, Chi TY, Xu TG, Ge SQ, Liu YT, Li JM, Wang SJ, Qu HL, Jin T, Wang ZL (2019) Genome comparison of African swine fever virus China/2018/ AnhuiXCGQ strain and related European p72 Genotype II strains. Transbound Emerg Dis 66:1167-1176

Bastos ADS, Penrith ML, Cruciere C, Edrich JL, Hutchings G, Roger F, Couacy-Hymann E, Thomson GR (2003) Genotyping field strains of African swine fever virus by partial p72 gene characterisation. Arch Virol 148:693-706

Chapman DAG, Tcherepanov V, Upton C, Dixon LK (2008) Comparison of the genome sequences of nonpathogenic and pathogenic African swine fever virus isolates. J Gen Virol 89:397-408

Costard S, Wieland B, de Glanville W, Jori F, Rowlands R, Vosloo W, Roger F, Pfeiffer DU, Dixon LK (2009) African swine fever: how can global spread be prevented? Philos Trans R Soc B Biol Sci 364:2683-2696

Dixon LK, Chapman DAG, Netherton CL, Upton C (2013) African swine fever virus replication and genomics. Virus Res 173:3-14

Dixon LK, Sun H, Roberts H (2019) African swine fever. Antiviral Res 165:34-41

Edgar RC (2004) MUSCLE: a multiple sequence alignment method with reduced time and space complexity. BMC Bioinform 5:1-19

Iyer LA, Balaji S, Koonin EV, Aravind L (2006) Evolutionary genomics of nucleo-cytoplasmic large DNA viruses. Virus Res 117:156-184
Jori F, Vial L, Penrith ML, Perez-Sanchez R, Etter E, Albina E, Michaud V, Roger F (2013) Review of the sylvatic cycle of African swine fever in sub-Saharan Africa and the Indian ocean. Virus Res 173:212-227

Medini D, Donati C, Tettelin H, Masignani V, Rappuoli R (2005) The microbial pan-genome. Curr Opin Genet Dev 15:589-594

Sanchez-Vizcaino JM, Mur L, Gomez-Villamandos JC, Carrasco L (2015) An update on the epidemiology and pathology of African swine fever. J Comp Pathol 152:9-21

Stamatakis A (2014) RAxML version 8: a tool for phylogenetic analysis and post-analysis of large phylogenies. Bioinformatics 30:1312-1313

Tcherepanov V, Ehlers A, Upton C (2006) Genome Annotation Transfer Utility (GATU): rapid annotation of viral genomes using a closely related reference genome. BMC Genom 7:150

Vernikos G, Medini D, Riley DR, Tettelin H (2015) Ten years of pangenome analyses. Curr Opin Microbiol 23:148-154

Wang N, Zhao DM, Wang JL, Zhang YL, Wang M, Gao Y, Li F, Wang JF, Bu ZG, Rao ZH, Wang XX (2019) Architecture of African swine fever virus and implications for viral assembly. Science 366:640-644

Wen XX, He XJ, Zhang X, Zhang XF, Liu LL, Guan YT, Zhang Y, $\mathrm{Bu} Z \mathrm{ZG}$ (2019) Genome sequences derived from pig and dried blood pig feed samples provide important insights into the transmission of African swine fever virus in China in 2018. Emerg Microbes Infect 8:303-306

Zhao YB, Wu JY, Yang JH, Sun SX, Xiao JF, Yu J (2012) PGAP: pan-genomes analysis pipeline. Bioinformatics 28:416-418

Zhao YB, Jia XM, Yang JH, Ling YC, Zhang Z, Yu J, Wu JY, Xiao JF (2014) PanGP: a tool for quickly analyzing bacterial pangenome profile. Bioinformatics 30:1297-1299

Zhu ZZ, Xiao CT, Fan YS, Cai ZN, Lu CY, Zhang GH, Jiang TJ, Tan YJ, Peng YS (2019) Homologous recombination shapes the genetic diversity of African swine fever viruses. Vet Microbiol 236:108380 